\title{
LOS INICIOS DE LA FORMACIÓN DE PROFESORES EN MÉXICO (1821-1921)
}

\author{
Luz Elena Lafarga Galván \\ Centro de Investigaciones y Estúdios Superiores \\ en Antropologia Social, México.
}

$\cos 80$

\section{OS INÍCIOS DA FORMAÇÃO DE PROFESSORES NO MÉXICO (1821-1921)}

\begin{abstract}
Resumo professores.

Palavras-chave: formação de professores, profissionalização.

\section{THE BEGINING OF THE TEACHERS'S TRAINING IN MEXICO (1821-1921)}

No México, a carreira do magistério tem uma longa tradição. Começou como uma arte e se profissionalizou ao longo do tempo. Neste artigo se apresenta uma síntese dos diversos espaços acadêmicos no âmbito dos quais o magistério foi formado. Essa formação começou com o sistema lancasteriano e continuou em academias e institutos, para culminar nas escolas normais. Nessas instituições foi o ensino da Pedagogia que delimitou as diferenças entre a arte de ser professor e sua profissionalização. Em termos metodológicos, este trabalho se relaciona com a proposta de Dominique Julia, de conhecer quais eram os saberes e costumes que tinham os

\begin{abstract}
In Mexico teacher's training has a long tradition. It started as an art, until it got the status of a profession. This article shows the different academic spaces where teachers were trained. It started with its instruction at the Lancastrian system, and then they studied at the academies and institutes until the Normal Schools were created. In these institutions the future teachers received lessons of pedagogy that makes the difference between the art of being a teacher and its professionalization. These are the axes that cross the article. The main ideas are related to the question that Dominique Julia exposes in order to find out which was the knowledge that teachers had in the past.

Key-words: teachers, training, professionalization.
\end{abstract}




\section{LOS INICIOS DE LA FORMACIÓN DE PROFESORES \\ EN MÉXICO (1821-1921)}

\section{Resumen}

En México la carrera del magisterio tiene una larga tradición. Se inició como un arte y se fue profesionalizando. En este artículo se presenta un recorrido por los diversos espacios académicos en donde el magisterio fue formado. Se inicia con su instrucción en el sistema lancasteriano y se continúa con academias e institutos hasta culminar con las escuelas normales. En estas instituciones fue la enseñanza de la pedagogía la que marcó la diferencia entre el arte de ser maestro y su profesionalización. Estos son los ejes que atravesaron al artículo. En cuanto a la guía teórica y metodológica, ésta se relaciona con la pregunta que plantea Dominique Julia para conocer cuáles eran los saberes y costumbres que tenía el maestro.

Palabras-clave: maestros y maestras, formación, profesionalización.

\section{LES DÉBUTS DE LA FORMATION DES ENSEIGNANTS AU MEXIQUE (1821-1921)}

\section{Résume}

Au Mexique, la carrier de masgistère a une longue tradition. II a commence comme un art et il a été professionnalisée. Cet article présente un parcours à travers divers domains universitaires oú les maitres ont été formé. II démarre avec son instruction dans le systéme lancasteriane et se poursuit avec les academies et les instituts, culminant avec les écoles normales. Dans ces institutions est l'enseignement de la pédagogie qui ont marquee la difference entre l'art d'être maître et sa professionnalisation. Ce sont les thèrmes qui a traverse l'article. Sur Le guide théorique et méthodologique, cela se rapporte à la question posée par Dominique Juliá de savoir queles étaient les connaissances et les coutumes qui avait les maîtres.

Mots-clé: eiseignants et enseignantes, formation, professionnalisation.

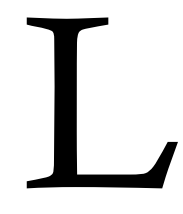
ugar común para el magisterio hoy en día, son las escuelas normales. De hecho, no se puede pensar en la formación de maestras y maestros, sin relacionarla con alguna escuela normal. Sin embargo ¿sucedía lo mismo en el pasado?, ¿cómo se formaban los maestros de ayer?, ¿en qué momento surgieron las escuelas normales en nuestro país?; éstas y otras dudas más son las que han guiado el presente artículo.

Para su realización, se han tomado en cuenta dos ejes: el del arte de ser maestra y maestro y el de su profesionalización, los cuales se inscriben en diversos espacios geográficos. De hecho, en cada uno de estos espacios geográficos, se construyen las instituciones educativas en diferentes tiempos y con sus propios ritmos.

En los primeros años del México independiente, se crearon algunos planteles escolares cuya finalidad era la de enseñar a los niños y a algunas niñas, a leer, escribir y hacer cuentas, como se decía coloquialmente. Sin embargo, poco a poco, los gobiernos locales se dieron cuenta de que era necesario formar a los futuros maestros y maestras 
con objeto de tener un control sobre la instrucción pública que se impartía, de aquí que se fundaran una serie de instituciones para cumplir con dicho objetivo.

Cabe recordar que no es sino hasta 1921 con la fundación de la Secretaría de Educación Pública, que se crea una instancia que se encargará de regir a la educación en todo el país, o sea a nivel nacional. De aquí que, anteriormente a esta fecha, cada estado manejara su sistema educativo de manera independiente y de acuerdo con el presupuesto que tuviera.

Esta aclaración permite explicar por qué los cien años que abarca este artículo se inician en 1821 con la vida independiente de nuestro país, y terminan en 1921 con la creación de la Secretaría de Educación Pública, ya que los historiadores de la educación han considerado a esta fecha como el final de una época y el inicio de otra.

\section{El arte de ser maestra y maestro}

Para explicar en lo que consistía este arte, es necesario introducirse en la Compañía Lancasteriana y sus escuelas normales. Esta Compañía se estableció en la Ciudad de México a principios de 1822. Se trataba de una asociación privada dedicada a implantar el nuevo método de la enseñanza mutua de los ingleses Joseph Lancaster y Andrew Bell. Sus escuelas eran fundadas por particulares además de ser gratuitas (Galván, 1985).

De acuerdo con este sistema, los alumnos más avanzados llamados monitores, previamente instruidos por el preceptor, o sea el maestro, enseñaban a grupos de 10 niños. La primera escuela que se fundó fue El Sol (1822) y pocos meses después Filantropía. En 1833 esta Compañía pudo cumplir un anhelo de muchos años: abrir su primera escuela para 160 niñas en Santa María la Redonda, uno de los barrios más pobres y abandonados de la ciudad. (Tanck, 1984, p.180-184). Así, las niñas también eran instruidas mediante este sistema. Los alumnos aprendían a escribir, ya no en pizarras como en otras escuelas, sino en cajas de arena en donde trazaban las letras con palitos.

En el sistema lancasteriano, si un alumno hacía un progreso más rápido en una asignatura que en otra, podría estar por ejemplo: en la segunda clase de escritura y en la tercera de lectura y en la primera de aritmética. El sistema no era graduado y podía haber chicos de distintas edades en diferentes clases ya que lo que determinaba su asignación era su habilidad y no su edad (Tanck, 1984).

Algo importante en esta escuela siguió siendo la memorización, como había sido en la época colonial, así como la obediencia, el silencio y el orden (Idem, 1984), y si no obedecían, venían los castigos. 
Poco a poco, se fue difundiendo por toda la República en donde había corresponsales en las capitales de los estados quienes eran los encargados de fundar escuelas y vigilarlas. José María Luis Mora, ideólogo del siglo 19, comenta que, aun cuando "la enseñanza primaria no se perfeccionó gran cosa, se difundió asombrosamente por toda la República" (Mora, 1949, p. 58). Por medio de este sistema se educaron en las asignaturas antes mencionadas, muchos mexicanos y algunas mexicanas. La Compañía Lancasteriana fue abolida hasta 1890 (Galván, 1985).

En cuanto a las Escuelas Normales de la Compañía Lancasteriana, en 1823 se fundó la primera en la ciudad de México en el ex-convento de Belén. Este sistema tenía la posibilidad de que, un solo maestro podría enseñar a 1.386 alumnos, divididos en tres aulas; de primeras letras; una especie de secundaria con clases de francés, latín, dibujo y matemáticas; y la Escuela Normal con un cupo para 418 alumnos.

Esta escuela abrió sus puertas en la segunda mitad de 1823, desgraciadamente su vida fue muy corta, debido a la falta de estudiantes. En febrero de 1824 se decía que la Compañía había advertido con dolor que "no se han presentado hasta ahora sino cinco o seis" (Tanck, 1984, p. 237). De aquí que exhortara de nuevo a los preceptores para que aprendieran el método en tan sólo seis meses. Las clases fueron impartidas en las noches durante cuatro meses, y dos más de práctica en una escuela para adultos.

En los estados, también se fundaron escuelas normales lancasterianas, como en Zacatecas, Veracruz y Chihuahua, en 1826; y en Tamaulipas, Jalisco, Michoacán y Chiapas, en 1828. Posteriormente, se fundó la de Nuevo León en 1842. De este modo, la primera institución que surgió en nuestro país para la formación de los futuros preceptores (profesores) para la enseñanza elemental, partió de una iniciativa particular, sin embargo contó con apoyo económico de los gobiernos de los estados que, además, les facilitaron antiguos ex conventos y otros edificios para establecer dichas escuelas.

Existe un estudio sobre la Normal Lancasteriana de Zacatecas. Su autor comenta que de todas las escuelas fundadas en dicho estado entre 1824 y 1835 que es el periodo de la primera República Federal, la que tuvo mayor importancia debido a su impacto fue la Normal de la Constitución. Se proponía la enseñanza mediante el método lancasteriano y capacitar a preceptores en servicio en todo el estado, para que regresaran a sus lugares de origen, establecieran escuelas y propagaran el nuevo método.

En sus primeros años funcionó como una primaria lancasteriana y no fue sino hasta 1831 que se transformó en institución formadora y actualizadora de preceptores en servicio. Tanto las escuelas lancasterianas como la Normal de la Constitución, tenían 
entre sus objetivos, preparar a los futuros ciudadanos con el fin de "hacerlos útiles al país y prepararlos a los goces de la vida social" (Contreras, 2005, p. 194).

La Compañía Lancasteriana cubrió un largo período, de 1822 a 1890. A pesar de que en su última etapa ya no fue tan efectiva, es importante reconocer su continuidad en medio de los problemas tanto políticos como económicos, que vivió nuestro país durante el siglo 19. Fue en esas escuelas y con el sistema de enseñanza mutua, que se formaron varios de los preceptores decimonónicos. Sobre este sistema, José María Luis Mora comentaba que no había logrado "fundar ni sostener el número de escuelas que había deseado, pero su avance había sido notable" (Mora, 1949, p. 65).

\section{En camino hacia la profesionalización del magisterio}

Al iniciarse la década de 1870, una vez concluido el Imperio de Maximiliano y al iniciarse el gobierno del presidente Benito Juárez (1867-1873), el magisterio mexicano llevaba una larga trayectoria. Su formación se había realizado por medio de las escuelas lancasterianas, o bien por medio de la práctica con otros maestros. Era como un oficio, como un arte de ser maestra y maestro. De aquí la importancia del inicio de esta profesionalización del magisterio.

Dominique Julia (1995, p. 143) se hace la siguiente pregunta en relación con la profesionalización de los docentes: “¿cuáles son los saberes y costumbres requeridos a un futuro enseñante? Es esta inquietud teórica y metodológica la que guiará este inciso".

Ahora bien, durante la República Restaurada y el Porfiriato, el magisterio "se expandió y creció cualitativamente", además de que "dejó de ser una profesión casi libre para convertirse en una profesión de estado". Las escuelas normales, entonces, se preocuparon tanto por las "materias pedagógicas", como por la forma de enseñarlas. Este hecho llevó a un cambio en su "identidad profesional" (Arnaut, 1996, p. 130-139). De este modo, las escuelas normales fundadas a partir del porfiriato e, incluso, algunas de ellas varios años antes, se empeñaron en que uno de los principales saberes debería de ser el de la pedagogía.

Durante el régimen de Díaz y sobre todo después de los Congresos de Instrucción Pública (1889 y 1890), se deseaba que el eje que estructuraría a los programas de las escuelas normales, fuera el de los cursos pedagógicos $\mathrm{y}$, en especial, aquellos de carácter práctico.

En el Segundo Congreso de Instrucción Pública (1890), se inició la discusión sobre el currículum de las escuelas normales, sin embargo como no hubo tiempo para ponerse de acuerdo sobre su distribución, se propuso "dejar a las escuelas normales la plena 
libertad de organizarse en consonancia con las necesidades y costumbres de las regiones en que estén llamadas a ejercer su acción fecunda". De hecho, se consideró que era de "absoluta necesidad la difusión del normalismo", por ello se creó, en cada entidad federativa, un centro que tenía la finalidad de propagar el magisterio.

Los maestros que fueran a trabajar en las primarias elementales ( $1^{\circ}$ a $4^{\circ}$ grado), cursarían tres años en la escuela normal, y los de la primaria superior $\left(5^{\circ}\right.$ y $6^{\circ}$ grado), tendrían que permanecer en ella cinco años. Se decidió que, para ingresar a la escuela normal, no era necesario haber cursado la preparatoria. Además de preocuparse por la preparación del futuro maestro, en este Congreso también se puso el acento en la conveniencia de que contaran con un título (Galván, 1980).

Durante el porfiriato, comenta González Navarro (1998), el número de escuelas normales aumentó de 12, en 1878, a 26, en 1907; tres eran de mujeres y se cuadruplicaron tres décadas después. Aclara que no todos los estados contaban con escuelas normales, ya que algunos tenían la carrera del magisterio pero se cursaba en los institutos o colegios. Se refiere también a otros estados que, como Baja California, Tlaxcala, Hidalgo, Morelos y Quintana Roo, "no dieron en ninguna forma esa enseñanza" (p. 667).

De hecho la fundación de estas escuelas normales fue muy irregular, ya que cada estado tenía sus propios tiempos y ritmos. De aquí que, en ocasiones se formara a los docentes por medio de secundarias, escuelas superiores, liceos o academias, Era frecuente que las escuelas normales formaran parte de algún instituto literario, o bien de algún colegio de educación superior (Galván, 1991).

De aquí que todas estas instituciones en donde se formaba el magisterio, se inscribieran dentro de este camino hacia la "profesión de estado". En este apartado se darán algunos datos de su fundación, de acuerdo con los datos obtenidos, lo cual no significa que no existieran en aquellos estados que no se mencionarán.

\section{Diversas escuelas para varones y para niñas}

a) La Secundaria de Niñas en la ciudad de México. El hecho de que, en la ciudad de México no hubiera escuelas normales, llevó a que se acostumbrara el obtener licencia para ejercer el magisterio, mediante exámenes presentados ante la Comisión de Instrucción del Ayuntamiento.

Poco a poco, la profesión del magisterio se convirtió en una opción de trabajo para la mujer. De este modo, en la Ley Orgánica de Instrucción Pública de 1867, se incluía la fundación de una escuela secundaria para el sexo femenino, que empezó a funcionar en 
1869. En 1878 cambió su nombre por el de Escuela Nacional Secundaria de Niñas, su plan de estudios se aumentó en seis años en lugar de cinco. Protasio Tagle incluyó en este nuevo plan de estudios, cursos de ciencias físicas y naturales, higiene, medicina, economía doméstica, repostería y pedagogía que impartía el doctor y pedagogo Manuel Flores.

En esta secundaria, además de formarse las futuras maestras, también se habían incluido asignaturas que constituían "una especie de carreras terminales independientes y diferentes de la del magisterio". Entre otras se encontraba la enseñanza de la telegrafía (González Navarro, 1985, p. 666).

b) Institutos para niñas. Fundación del Instituto de Niñas en Durango. Este Instituto se fundó en 1870. En él, se estudiaba para ser Profesora de Instrucción Primaria, también se impartían estudios comerciales y de telegrafista. Las niñas que deseaban llegar a ser profesoras, deberían tener una edad mínima de 11 años. Las materias que se cursaban eran las siguientes: tejidos, bordados, flores y música, todas estas materias las impartían varias maestras; y las de gramática castellana, francés, aritmética y dibujo, impartidas por maestros. Como se ve por las materias impartidas, hasta ese momento todavía no se estudiaba la de pedagogía

En enero de 1876, el gobernador expidió una Ley de Instrucción Pública en donde se establecía que la enseñanza pública en ese estado se dividiría en: Primaria, Secundaria y Profesional. También se decía que para la enseñanza profesional se establecerían las siguientes escuelas especiales: Escuela Normal, Jurisprudencia, Ingenieros, Medicina y Artes.

Se especificaba que el objetivo de la Escuela Normal era el de la formación de preceptores. Se decía que en ella se impartirían cátedras para hombres y para mujeres. También existían preceptores de primeras letras de $1^{a}, 2^{a}$, y $3^{a}$ clase, según la instrucción que para cada caso fijara la ley. De aquí que el Instituto de Niñas siguiera funcionando, pero a partir de ese momento se le otorgaba el carácter de escuela normal (Guzmán, 1987, p. 10).

Es interesante hacer notar que, cuando al Instituto de Niñas se le dio la categoría de escuela normal, cambió su plan de estudios. Aparecieron dos materias importantes que, de hecho sólo cursaban los maestros de $1^{\mathrm{a}}$ y $2^{\mathrm{a}}$ clase, que eran las de conocer los métodos que se utilizaban en Europa y América y la de la enseñanza objetiva.

Se puede decir que éste fue el inicio de las clases de pedagogía ya que no hay que olvidar que el origen de su desarrollo en México es europeo. Así el conocimiento de estos métodos, permitía que el futuro maestro se fuera introduciendo en diversas teorías 
pedagógicas. Una de ellas, que en ese momento estaba de moda en Europa era la que en Alemania y Francia se conocía con el nombre de lecciones de cosas, y en Inglaterra y Estados Unidos, con el de enseñanza objetiva.

De este modo, nos encontramos con otro momento dentro de la formación del magisterio, en donde los maestros tenían que aprender un método de enseñanza el cual era necesario que practicaran. De aquí la importancia de las prácticas pedagógicas que realizaban en algún momento de su carrera (Meneses, 1983).

En 1891 se expidió en Durango la Ley Orgánica y Reglamentaria de Instrucción Pública en la que, en el artículo 136 se decía que los alumnos que desearan obtener el "Título de Profesor(a) de Instrucción Primaria cursarán la clase de Pedagogía y Métodos de Enseñanza", al concluir todos sus estudios, o al ser aprobados en el $4^{\circ}$ año, "practicarán un año en alguna escuela primaria cuando menos 2 horas diarias" (Guzmán, 1987, p. 32).

Al avanzar el porfiriato, se refuerza la idea de inculcar la pedagogía en los futuros maestros y maestras y de que cumplieran con sus prácticas.

c) Liceos para $v$ arones y para niñas. El Liceo de Varones en Colima. Abrió sus puertas por primera vez en 1874 y tuvo que clausurarse en 1884, después de una epidemia de fiebre amarilla. Este Liceo fue una Institución Superior Secundaria que se había fundado en lugar del Colegio Civil (1859). Aquí se preparaba a los futuros maestros, quienes recibían el título de Preceptores de Primero, Segundo o Tercer Orden, de acuerdo con la eficiencia de los sustentantes. Uno de los maestros mexicanos que fueron muy reconocidos durante el siglo 19, Gregorio Torres Quintero, estudió en este Liceo, en donde obtuvo el título de Preceptor de Primer Orden.

En 1884, se fundó la Escuela Normal de Señoritas, en donde se preparaba a las preceptoras y también se les asignaba el título de Preceptora de Primero, Segundo o Tercer Orden. En 1887 el gobierno del Estado suprimió esta escuela normal, por lo que las escuelas elementales fueron las encargadas de preparar a las alumnas en el arte de ser maestra, siempre y cuando cumplieran con el programa de estudios que había regido en la escuela normal, quienes no se titulaban sólo podían trabajar como maestras en las escuelas rurales (Hernández, 1961, p. 77).

d) El Liceo de Niñas en Aguascalientes. Este Liceo se fundó en 1878, ya que existía una preocupación por formar a las maestras de la niñez que vivía en dicho estado. Sin embargo se dice que el principal objetivo era el de ofrecer educación a las señoritas, de acuerdo con las influencias extranjeras que llegaban de Inglaterra, Alemania, Francia y 
Estados Unidos. Esta institución estaba inspirada por los ideales de orden y progreso e influida por el pensamiento positivista de la época.

Una de sus características era que los maestros no devengaban un salario ya que su colaboración era gratuita. Su dirección quedó en manos femeninas, se decía que debería de ser una señora de mayor edad, disfrutar de una reputación bien sentada y no tener hijos pequeños, era nombrada por el gobernador ante quien la Junta de Instrucción hacía sus propuestas. En 1888, se fundó la cátedra de telegrafía, con objeto de que las alumnas que no terminaran sus estudios de educación normal, tuvieran una preparación que les permitiera conseguir un empleo.

Desde su creación, recibió todo el apoyo del gobierno del estado. En el año de 1915 se transformó en Escuela Normal del Estado, pero siguió formando solamente a mujeres dentro del magisterio. En un principio se le presionó para que dejara de ser católica, sin embargo pronto se distanció de la ideología revolucionaria para regresar a la conservadora (Barba y Zorrilla, 1993, p 15-17).

\section{Academias e institutos científicos y literarios}

a) Academias en la ciudad de México. Las academias jugaron un papel muy importante tanto en la formación del magisterio, como posteriormente en su profesionalización, sobre todo en aquéllas regiones en donde no se habían creado escuelas normales.

Desde los primeros años del México independiente, existió esta clase de academias en la capital de la República Mexicana. En 1827 se formó una Academia de Primera Enseñanza organizada por Ignacio y Juan Espinosa de los Monteros, padre e hijo, quienes se proponían uniformar la enseñanza de la primera edad. A su inauguración asistió el presidente Guadalupe Victoria. Posteriormente, la Secretaría de Justicia e Instrucción Pública acordó, en septiembre de 1879, la creación de las Academias de Instrucción Primaria, en las cuales se actualizaría el magisterio.

Por medio de ellas se trataba de uniformarlos en métodos modernos de enseñanza, a través de clases de pedagogía e intercambio de experiencias. Para ello, se reunirían dos veces al mes. En 1882, la Comisión de Instrucción Pública estableció la Academia de Pedagogía, formada por los profesores de las escuelas municipales mixtas la cual se ocuparía del estudio del sistema objetivo y oportunamente, de los demás ramos que comprende.

Las sesiones se llevaban a cabo todas las tardes durante una hora y se alternaban un día las profesoras y, otro, los profesores y su asistencia era obligatoria. Su presidente 
fue el pedagogo Luis E. Ruiz. Su método consistía en designar a un profesor/a para que disertara y pusiera a debate algún tema relacionado con la enseñanza objetiva. Con ello se proponían combatir la memorización y la enseñanza de la lectura y escritura con base en el deletreo, así como actualizar a los profesores quienes, por falta de una escuela normal, carecían de una formación pedagógica (Meneses, 1983).

b) La Academia Normal de Veracruz. En 1885, se fundó una Academia Normal la cual se estableció en la llamada Escuela Modelo de Orizaba, Veracruz. El profesor Enrique Laubscher tuvo a su cargo la dirección práctica, además de que impartía las clases de aritmética, geometría y dirigía la práctica escolar; y el profesor Enrique C. Rébsamen tuvo a su cargo la dirección científica y teórica, además de que enseñaba pedagogía, inglés y francés. De los 18 cantones, llegaron 22 estudiantes que eran, "maestros empíricos ameritados". El curso duró 6 meses y, después, regresaron a sus lugares de origen para dirigir las escuelas cantonales (Reyes, 1959, p. 88-100).

c) La Academia de Toluca. En 1893, se estableció la Academia Pedagógica de la Municipalidad de Toluca en el Estado de México, con objeto de facilitar la comunicación de las ideas y principios pedagógicos entre los profesores de las escuelas primarias, también se proponían discutir los procedimientos metodológicos y uniformar los "métodos de enseñanza de las escuelas primarias. En 1897, la Ley de Instrucción Primaria estableció que en cada municipalidad debería existir una Academia Pedagógica, integrada por todos los profesores/as, que sesionaría todos los sábados (García Luna, 1994, p. 37).

d) Academias de Varones y Señoritas y la Escuela Normal de Preceptores en Sinaloa.

A manera de antecedente, se puede mencionar la fundación, en 1874, de las academias adjuntas de varones y señoritas, cuyo plan de estudios contenía las mismas asignaturas que la instrucción primaria de segunda clase. Posteriormente, en el año de 1881, estas academias se transformaron en la Escuela Normal de Preceptores al interior del Colegio Rosales. La carrera duraba dos años, y para estudiarla no se necesitaba haber cursado la preparatoria. Entre las materias que impartían se menciona el curso de pedagogía (Sánchez, 1998, p. 108-111).

e) El Instituto Literario de Toluca y la Escuela Normal Para Profesores. En el estado de México, la Escuela Normal para Profesores se fundó, provisionalmente, en el Instituto Literario de Toluca en el año de 1882. Ahí se formaron los preceptores que prestaban sus servicios en las escuelas de primera, segunda y tercera clase. 
Esta Normal continuó anexa al Instituto hasta febrero de 1903 en que, por decreto, se emancipó de él y se le otorgó toda la parte antigua del Instituto Literario, con una organización independiente. Esta Normal tuvo su edificio propio hasta septiembre de 1910, el cual se construyó de acuerdo con los conceptos de la moderna pedagogía (García Luna, 1994, p. 37).

f) El Instituto Literario de Chihuahua y la formación del magisterio. En junio de 1885 se declaró oficialmente establecida por el Gobierno la Cátedra de Pedagogía en el Instituto Literario. En el año de 1886, la enseñanza objetiva que había impulsado Rébsamen, se convirtió en la nueva metodología que los maestros tenían que aprender y practicar. De aquí que, a partir de este año a los maestros se les exigiera su asistencia a la clase de pedagogía.

Hacia 1894, la escula normal ya estaba integrada al Instituto Científico y Literario y así funcionó hasta su separación en 1937 .En el año de 1905 se reglamentó la creación de la Escuela Normal Mixta, mediante la Ley de Enseñanza Normal del Estado y se expidió el Reglamento de Conferencias Pedagógicas. En 1906 cuando abrió sus puertas esta escuela normal, en el estado de Chihuahua había

45 maestros normalistas titulados, 30 con estudios terminados pero sin título y 310 sin estudios de normal."Esta escuela inició sus trabajos como una parte del Instituto Científico y Literario y, por lo tanto, en el mismo edificio que ocupaba dicho Instituto. (Hernández Orozco, 1999, p. 161)

g) El Instituto Científico y Literario de Tlaxcala y la formación del magisterio. Como antecedentes en la formación del magisterio, se pude mencionar el establecimiento del Colegio Normal del Estado, en 1882. En él se preparaban, por un lado, los maestros que prestaban sus servicios en escuelas urbanas, ya que se les ofrecía la pedagogía didáctica en toda su ex tensión.

Por otro lado, también acudían maestros rurales que se encontraban en servicio, y que ahí podrían ampliar los ramos de la primaria además de que obtendrían elementos de pedagogía y de solfeo. Hacia 1893, el gobernador ordenó que todos los directores de escuelas fueran a dicho Colegio con la finalidad de que cursaran materias pedagógicas por un tiempo conveniente.

Posteriormente, el Instituto Científico y Literario fue el encargado de formar a los futuros maestros. En cuanto a las maestras, se formaban en la Escuela Superior de Niñas, ambas instituciones se fundaron en 1897. Contaban con un programa de becas, por medio del cual se sostenían los estudios de primaria superior de los becarios y becarias. Quienes no pudieran continuar un año más los estudios pedagógicos, prestaban 
sus servicios en las primarias elementales de la región. De hecho, al igual que el Colegio Normal, lo que se ofrecía era un año posprimario de pedagogía, influencia que había recibido de la Escuela Normal de Jalapa en Veracruz.

La Ley de Educación Popular de 1917, ofrecía a los maestros en servicio un Curso de Normal con derecho a obtener un título después de tres años de estudios. Este título tendría la fuerza de un título normalista. Sin embargo, pocos eran los maestros que ejercían con título, como lo demuestra una encuesta realizada a 214 maestros en 1918 , de los cuales sólo 29 tenían título de normalista (Rockwell, 1996).

\section{Escuelas normales}

a) Escuelas Normales en San Luis Potosí. La primera Escuela Normal para Varones, se fundó en el año de 1848 y estuvo a cargo del señor Vallejo, quien era el director de la escuela lancasteriana y además tenía permiso para la enseñanza particular en su casa. Posteriormente el gobernador lo apoyó para la creación de Escuela Normal. Los primeros alumnos normalistas potosinos fueron cuatro jóvenes, que vivían en la ciudad de San Luis Potosí y diez, que habían llegado de los pueblos vecinos.

En el reglamento de la Escuela Normal se establecía el compromiso de los alumnos de corresponder a lo que el gobierno había invertido en su educación. Por ello, deberían trabajar para las escuelas públicas del estado durante cinco años, por lo menos. En cuanto a la creación de la Escuela Normal para Señoritas, ésta se anunció al mismo tiempo que la de Varones, sin embargo no se fundó sino hasta el año de 1868.

Entre los requisitos se solicitaba que los hombres tuvieran entre 17 y 25 años y las mujeres entre 12 y 16. Al ingresar a la Escuela Normal, las mujeres no cursaban gramática avanzada, ni geometría ni dibujo lineal, materias que sí se impartían a los varones. En cambio, cursaban economía doméstica, bordado y corte de ropa interior. Tanto hombres como mujeres, podían optar por ser maestros de primero, segundo o tercer orden. Posteriormente, en la Ley de Instrucción Primaria de 1885, se decía que la carrera en ambas escuelas normales debería realizarse en seis años, y entre las materias que estudiaban se encontraba la de pedagogía, desde el primer año de estudios (Muro, 1899).

b) Escuelas Normales en Guanajuato. En 1869 se decretó oficialmente la creación de dos Escuelas Normales; una para Señoritas y otra para Varones, las cuales abrieron sus puertas en 1871. A partir de esa fecha, la formación del magisterio en estas normales duraba dos años, en 1873 los estudios se ampliaron a tres y para 1878 se deberían cursar cinco años. 
Además del número de materias, también se aumentó un año más de prácticas. Los cursos eran teórico-prácticos. Entre algunos se pueden mencionar los de ortología, caligrafía, aritmética, gramática, derechos y deberes civiles, lecciones de moral, urbanización, sistema de enseñanza mutua y construcción de útiles escolares. En la Normal para Señoritas, además, se enseñaba economía doméstica, corte de ropa blanca interior, corte de vestidos para niños y señoras, y bordados (Archivo Histórico de la Alhóndiga, caja 180-A).

Para ocupar la dirección, era necesario realizar un concurso de oposición. Una de sus directoras, la profesora Angelina Castelazo quien había egresado de dicha institución afirmaba que aun cuando las materias eran pocas, éstas se estudiaban a conciencia. Sus prácticas las realizaban en las escuelas municipales (Archivo Municipal de Guanajuato, 1875/05/20).

Ambas Normales contaron con becas para alumnos y alumnas pobres. De aquí que en la documentación existan constancias en donde se acreditaba la "buena conducta y suma pobreza" de quienes aspiraban a obtener una beca para poder continuar con sus estudios. En las cartas también se decía que se trataba de jóvenes que vivían en las áreas rurales (Archivo Municipal de Guanajuato, 1878/01/16, exp. 835 y 836).

Con el tiempo, desapareció la Escuela Normal para Varones. Por esta razón, la formación de maestros se impartía en el Colegio del Estado. En 1885, durante el régimen de Porfirio Díaz se fundó una Normal Mixta.

c) Puebla y su Escuela Normal. En este estado, el Congreso aprobó la fundación de la Escuela Normal para Señoritas, el 15 de septiembre de 1879. Su primera directora fue la maestra y poetisa Paz Montaño, a quien el Ayuntamiento de la Ciudad de México le había expedido su título de profesora. En cuanto a la Escuela Normal para Varones, ésta se fundó el 4 de enero de 1880, y su primer director fue un distinguido intelectual mexicano: Guillermo Prieto. Estas dos Normales se unieron el 16 de marzo de 1906, con el nombre de Instituto Normal del Estado. Entre una de las materias que se ofrecían, se encontraba la de pedagogía didáctica. (García, 1940, p. 15-25).

d) La Escuela Normal de Artes y Oficios para Señoritas en el estado de México. Esta escuela se fundó en 1891 como resultado del Asilo de Niñas y de la escuela José Vicente Villada. Tenía varias secciones: Párvulos, Primaria, Secundaria, Maestra de Artes y Oficios y Profesora. Las alumnas que quisieran obtener el titulo de Profesoras de Instrucción Primaria de Primera Clase, deberían estudiar durante cinco años.

Llevaban cursos de pedagogía teórico práctica, metodología y legislación de instrucción primaria en el Estado y en el cuarto año, realizaban sus prácticas pedagógicas 
en la sección primaria. Para las profesoras de Instrucción Primaria de Segunda Clase, se les exigía solamente tres años de estudios. Posteriormente, se dictó una disposición con objeto de que, cada Distrito enviara a una alumna indígena, para que estudiara durante dos años y recibiera el título de Profesora de Instrucción Primaria de Tercera Clase. Uno de los requisitos era el que las alumnas regresaran a sus lugares de origen para ejercer el magisterio. Durante estos dos años, también recibían cursos de pedagogía y realizaban sus prácticas pedagógicas.

Otras escuelas normales que también se fundaron en el país entre 1880 y 1900 fueron las de Oaxaca, Querétaro, Tamaulipas, Jalisco, Michoacán, Guerrero, Colima, Coahuila y Nuevo León, entre otras. Muchas de ellas iniciaron como Academias para niñas, o bien para maestras y/o maestros y, poco a poco, se fueron transformando en las Escuelas Normales de la entidad. Sin embargo, la Escuela Normal que sirvió de ejemplo para la formación del magisterio en todo el país fue la de Jalapa, en Veracruz.

e) La Escuela Normal para Profesores en Jalapa, Veracruz. Esta Normal se fundó en 1886. Sus cursos duraban tres años para los profesores de instrucción primaria elemental y cinco, para los de instrucción primaria superior. El Ejecutivo pensionaba a siete alumnos que estudiaban para profesor de primaria superior. Se podían inscribir todos aquéllos alumnos que cumplieran con los requisitos que marcaba su reglamento.

Sin embargo, se insistía en que " en igualdad de aptitudes entre dos o más aspirantes, se preferirá siempre el más pobre, y si en ellos concurriesen las mismas circunstancias de aptitud, dedicación y pobreza, la suerte designará al agraciado" (Zilli, 1996, p. 25).

Para poder ingresar se tomaba en cuenta la edad de quince años en adelante; el que tuviera "buenas costumbres y moralidad reconocida", lo cual se demostraba con un certificado del Alcalde Municipal del lugar de su residencia; que contara con la instrucción primaria elemental, para lo cual presentaba un examen ante el Director de la Escuela Normal; que tuviera "buena salud y estuviera vacunado", por lo que debía presentar un certificado de un médico titulado; y "comprobar legalmente que no tenía defectos orgánicos que dificultaran el desempeño del magisterio". (Reyes, 1959, p. 88-90).

Entre algunas de las materias que se impartían durante los tres primeros años se puede mencionar la de pedagogía, la cual comprendía didáctica, metodología, disciplina y legislación escolar. Los profesores de instrucción primaria superior, deberían de estudiar, entre muchas otras materias, la de pedagogía e historia de la pedagogía. Además, en todos los años de ambos cursos, se realizaban "ejercicios prácticos" en la escuela primaria anexa a la Normal; y los alumnos de tercero a quinto, visitaban otras escuelas 
primarias del estado. González Navarro, comenta que desde "los ochenta se establecieron cátedras de pedagogía en los colegios preparatorios y en las escuelas primarias superiores veracruzanas" (González Navarro, 1985, p. 670).

Cabe mencionar que esta Escuela Normal de Profesores de Jalapa, Veracruz, tuvo mucha importancia en la historia de las escuelas normales durante el siglo 19 ya que, su director Enrique Rébsamen influyó, con sus ideas sobre las asignaturas de pedagogía y metodología y su aplicación en la escuela práctica anexa, en la organización de las escuelas normales de Oaxaca, Guanajuato, Jalisco y en la estructuración de la Escuela Normal de la ciudad de México.

También intervino en los Congresos Nacionales de Instrucción Pública (1889-1890). Por otro lado, algunos de sus alumnos dirigieron escuelas, tanto en el estado de Veracruz, como en varios Estados, difundiendo así las principales ideas de la pedagogía moderna.

f) Las Escuelas Normales en la ciudad de México. La fundación de la Escuela Normal para Profesores en la capital de la República, fue tardía. De hecho, se habían realizado varios intentos por formar escuelas normales, pero ninguno de ellos había dado resultado.

De este modo, no fue sino hacia 1884 , se elaboró un proyecto para la creación de la Escuela Normal en la ciudad de México en donde se distinguía por su gratuidad y por su carácter nacional ya que no se limitó a recibir alumnos del DF, sino también de los Estados. Este fue un rasgo que se arraigó en la concepción de esta normal, que abrió sus puertas el 24 de febrero de 1887 bajo la dirección del profesor Miguel Serrano, en el ex convento de Santa Teresa. Contaba con dos escuelas anexas: una de párvulos y otra de primaria (Galván, 1991, p. 192).

El plan de estudios, ya reformado, comprendía cuatro años, estructurados con base en 8 grandes áreas, en donde la más importante se centraba en la formación profesional teórica y práctica de los alumnos quienes, durante los cuatro años cursaban historia de la pedagogía, sistema Froebel y pedagogía, además de la práctica empírica de los métodos de enseñanza en las escuelas anexas.

El plan de estudios muestra una institución en donde el acento estaba puesto, en la teoría pedagógica y en su práctica, principalmente. Se trataba de formar al maestro moderno, al maestro que no sólo conociera de teoría sino que también supiera cómo aplicarla frente a un salón de clases. Este será un principio importante dentro de la formación del magisterio en país.

En el año de 1888, se expidió el decreto que disponía la transformación de la Escuela Secundaria de Niñas, en Escuela Normal de Profesoras de Instrucción Primaria. 
Esta institución abrió sus puertas el $1^{\circ}$ de febrero de 1890 , bajo la dirección de la profesora Rafaela Suárez, en el mismo edificio que ocupaba la Secundaria de Niñas (exconvento de la Encarnación), y junto a ella se encontraba una primaria y una escuela de párvulos.

Durante el discurso de inauguración, el profesor Miguel Serrano decía que esperaba que "así como el siglo 18 dio al hombre la libertad por medio de la educación, el 19 diera a la mujer la igualdad por medio de ella". El currículum de esta Normal era el mismo que en 1878, sólo que ahora la carrera se cursaría en cuatro años, en lugar de seis, y las materias que en la Secundaria de Niñas se cursaban para algunas "carreras terminales" se eliminaron, quedando en su lugar un "curso opcional de conocimientos útiles" (Jiménez Alarcón, 1979, p. 120).

Durante el periodo revolucionario, maestras y maestros de la ciudad de México se enfrentaron a un grave problema ya que, en 1917 Venustiano Carranza suprimió la Secretaría de Instrucción Pública y Bellas Artes, con lo cual quedó la educación en manos de los ayuntamientos. Esta medida en nada ayudó al mejoramiento de la instrucción pública y menos al de los maestros.

Para 1919 había ya dos mil maestros de la capital declarados en huelga, debido a que no se les había pagado durante meses. Grupos de obreros y estudiantes se solidarizaron con ellos. Sin embargo, nada de esto sirvió y, finalmente, los maestros fueron cesados en masa. Este problema indicaba la necesidad de que existiera una Secretaría de Educación Pública a nivel nacional, la cual se creó en el año de 1921, lo cual marca otro momento dentro de la historia de la educación pública en nuestro país. Por ello en este artículo me detengo en esta fecha.

\section{Una reflexión final}

El abanico que se abre al introducirnos en el mundo de la formación de las maestras y de los maestros de ayer demuestra, por un lado, que en México se puede hablar de una importante tradición magisterial y, por otro, que existen muchas líneas de investigación en derredor de esta temática.

Una de estas líneas es el estudio de las ciudades como generadoras de espacios educativos, entre otros las Escuelas Normales. Las ciudades, brindaron oportunidades de trabajo a hombres y mujeres, y no sólo a quienes habitaban en ellas, sino también a quienes llegaban de las áreas rurales. Al fundarse las Escuelas Normales, las ciudades se convirtieron en lugares de trabajo para la industria, la minería, los servicios y el magisterio. 
Un ejemplo lo tenemos en la ciudad de San Luis Potosí en donde, desde la fundación de la Escuela Normal en 1848, la mayoría de sus alumnos procedían del medio rural. Posteriormente, la Normal de la ciudad de México se fundó con carácter nacional, con la idea de que a sus aulas pudieran llegar alumnos de otras regiones. Por ello, esta ciudad empezó a controlar la formación del magisterio en el ámbito nacional.

Hombres y mujeres, por igual, Ilenaron las aulas de las nuevas Escuelas Normales fundadas en diversas ciudades de la República Mexicana. Se les ofrecía, así, un empleo remunerado al terminar sus estudios. Algunos se quedaban en las mismas ciudades, pero otros más se iban a las áreas rurales, con lo que las ciudades empezaron a dar muestras de su poder en la definición de la educación, no sólo en el ámbito regional, sino incluso nacional.

Los hombres llevaban ya un largo tiempo en las aulas, sin embargo, para las mujeres fue una oportunidad de trabajo. En ocasiones estas mujeres salieron de sus hogares rurales y emprendieron la aventura hacia las ciudades, en donde estudiaron la carrera del magisterio. Para ellas, fue una gran oportunidad, no sólo porque dejaban el hogar paterno, sino porque llegaban a la gran ciudad.

Por lo general, estas mujeres se trasladaban muy jóvenes ya que tenían entre doce y trece años. Al llegar a las ciudades, las escuelas normales las protegían por medio de internados y becas para que pudieran continuar con sus estudios. Al egresar, se les ofrecía un trabajo remunerado, en una época en donde todavía no existía la igualdad entre hombres y mujeres.

Sin embargo, en el magisterio su labor empezó a ser aceptada, principalmente en las ciudades en donde existía una mayor apertura ideológica, que la que existía en el campo. Así, fue en las ciudades y no en las áreas rurales, en donde surgieron las primeras escuelas normales para las mujeres, las cuales siempre tuvieron una gran demanda, como sucedió en la Escuela Normal para Señoritas de la ciudad de México.

Otra línea que se perfila a través de este estudio, es la que se relaciona con la recuperación de la historia regional y la necesidad de contar con nuevas fuentes que permitan ampliar el panorama de la formación de los y las docentes en varios estados de la República Mexicana. Para lograrlo, es necesaria la formación de equipos de trabajo los cuales, desde sus propias regiones, se preocupen por el cuidado de sus acervos, tanto archivos como bibliotecas, y por la publicación de investigaciones que permitan avanzar en el conocimiento de este importante tema.

En este artículo se dio un breve panorama de cómo el magisterio ha transitado del arte ser maestra y maestro, a su profesionalización. Se trata de un camino largo en donde 
diversos hombres y mujeres empeñaron sus esfuerzos para cumplir, finalmente, sus expectativas.

Fue así cómo para llegar a la profesionalización se necesitaban determinados "saberes y costumbres" como apunta Dominique Julia (1995, p. 45). En México, los saberes se centraron en el conocimiento de la nueva pedagogía, de aquélla en donde la enseñanza objetiva desplazaba a la memorización.

En lo que se refiere a las costumbres, en el Segundo Congreso de Instrucción Pública se dejaba en libertad para que cada estado organizara sus escuelas normales de acuerdo a sus necesidades y costumbres. De aquí que no exista una homogeneidad sino que, más bien, se trate de una heterogeneidad que es la que caracteriza este proceso.

De hecho, los espacios geográficos que se revisaron tienen diferentes tiempos y ritmos que, en ocasiones, se cruzan. Se puede afirmar que este proceso fue muy irregular, ya que existieron diversas modalidades de instituciones que respondieron a las costumbres de cada estado, como por ejemplo: institutos de niñas, liceos, academias, e institutos literarios, entre otras más. Se trata de una serie de instituciones que, en ocasiones, sirvieron como base para la creación de las escuelas normales. Sin embargo, no siempre se siguió el mismo camino.

De aquí que la construcción de una historia de las escuelas normales no sea una tarea fácil. De hecho, se puede imaginar como la realización de un gran rompecabezas, en donde hay que juntar diversas piezas. La historia que se presentó en este artículo representa un primer intento por elaborar este gran rompecabezas y por responder a las preguntas planteadas al inicio del mismo. Pero también se espera que sea una provocación y un llamado para que esta historia se continúe construyendo desde las diversas regiones de nuestro país.

\section{Referências}

ARNAUT, Alberto. Historia de una profesión: los maestros de educación primaria en México, 1887-1994. México: Cide, 1996.

BARBA, José Bonifacio; ZORRILLA, Margarita. Las instituciones de educación normal en Aguascalientes. México: Universidad Autónoma de Aguascalientes, 1993.

CONTRERAS Betancourt, Leonel. Escuelas lancasterianas de Zacatecas en la primera República Federal, 1823-1835. México: Universidad Pedagógica Nacional, 2005.

GALVÁN, Luz Elena. Papel del maestro durante el porfiriato. In: Simposio sobre el magisterio nacional. México: Cisinah, 1980.

GALVAN, Luz Elena L. Los maestros y la educación pública en México. México: Ciesas, 1985. 
GALVÁN, Luz Elena L. Soledad compartida: una historia de maestros, 1908-1910. México: Ciesas, 1991.

GALVÁN, Luz Elena L. Del arte de ser maestra y maestro a su profesionalización. In: Diccionario de Historia de la Educación en México. Versión multimedia. México: Ciesas, Conacyt, DGSCA-Unam, 2002.

GARCÍA Luna, Margarita. La Escuela Normal de Profesores de Toluca. México: Gobierno del Estado de México, 1994.

GONZÁLIEZ Navarro, Moisés. El porfiriato: la vida social. In: VILLEGAS, Daniel Cosió. Historia moderna de México. México: Hermes, 1985, p. 495-526.

GUZMÁN, Noemí. Historia de una escuela. Durango: Gobierno del Estado, 1987.

HERNÁNDEZ, Francisco. Historia de la educación en el Estado de Colima. México: Gobierno del Estado de Colima, 1961.

HERNÁNDEZ Orozco, Guillermo. El Instituto Científico y Literario de Chihuahua, 18501900. México: Universidad Autónoma de Chihuahua, 1999.

JIMÉNEZ Alarcón, Concepción. Historia de la Escuela Nacional de Maestros. México: Secretaría de Educación Pública, 1979.

JULIA, Dominique. La cultura escolar como objeto histórico. In: MENEGUS, Margarita. Historia de las universidades modernas en hispanoamérica: métodos y fuentes. México: Unam, 1995, p. 131-153.

MENESES, Ernesto. Tendencias educativas oficiales, 1821-1910. México: Porrúa, 1983.

MORA, José María Luis. El clero, la educación y la libertad. México/DF: Empresas Editoriales, 1949.

MURO, Manuel. Historia de la instrucción pública en San Luis Potosí. México: Imprenta M. Esquivel y Cía., 1899.

REYES Rosales, José Jerónimo. Historia de la educación en Veracruz. México: Museo Veracruzano de Pedagogía, 1959.

ROCKWELL, Elsie. Hacer escuela: transformaciones de la cultura escolar, Tlaxcala 19101940. Tesis Doctoral en Investigación Educativa. México: DIE-Cinvestav, 1996.

SÁNCHEZ GASTÉLUM, José Luis. Educación y sociedad en Sinaloa: el Colegio Rosales, 1874-1918. Tesis Doctoral en Educación. México: Universidad Autónoma de Aguascalientes, 1998.

TANCK DE ESTRADA, Dorothy. La educación ilustrada, 1786-1836. México: El Colegio de México, 1977.

ZILLI, Juan. Historia de la Escuela Normal Veracruzana. México: Citlatepetl, 1996.

\section{Archivos}

Archivo Histórico de la Alhóndiga de Granaditas, caja 180-A. Reglamento de las Escuelas Normales del Estado de Guanajuato en 1873, México: Impreso por A. Chagoyán, 1873.

Archivo Municipal de Guanajuato, 1875/05/20 y Memoria del Centenario de la Escuela Normal de Guanajuato, 1971, p. 9 a 12.

Archivo Municipal de Guanajuato 1878/01/16, expedientes: 835 y 836. 
LUZ ELENA GALVÁN LAFARGA é professora no Centro de Investigaciones y Estúdios Superiores em Antropologia Social - Ciudad de México.

Endereço: Juarez, 87 - Centro de Tlalpan - Delegación Tlalpan - México - DF. E-mail: luzelena.galvan@gmail.com.

Recebido em 10 de março de 2012.

Aceito em 17 de maio de 2012. 